\title{
School-Based Suicide Prevention Strategy: Evidence-Based Data and Challenges
}

\author{
Yong-Sil Kweon \\ Department of Psychiatry, The Catholic University of Korea College of Medicine, Seoul, Korea
}

Despite international cooperation and national efforts to reduce the suicide rate, the effect on suicide prevention has not been so promising. In terms of society's resource availability and cost-effectiveness, it is reasonable to establish a suicide prevention policy that focuses on the high-risk group, which is expected to reduce the suicide rate. In South Korea, which has had the highest suicide rate among the OECD countries for over a decade, suicide prevention policies and strategies have focused on adults and the elderly population, both of whom have been identified as high risk for suicide [1]. In this context, preventing suicide among youth, who show a lower suicide rate than adults, has been implemented in school settings by the Ministry of Education (MOE) instead of the Ministry of Health and Welfare (MOHW), which is the main agency for health policies. Since school is a place where students spend most of their time and a protective environment in which we can apply various structured, non-academic student supports, school-based strategies for mental health promotion and suicide prevention can be actively implemented. The World Health Organization (WHO) has stressed the importance of school-based interventions as evidence-based interventions and is in the process of developing the Helping Adolescents Thrive (HAT) intervention package [2]. In South Korea, a nationwide school-based mental health screening test and mental health risk level-based support services are provided through the school system, local offices of education, and the MOE. Furthermore, the Suicide and School Mental Health Institute [3], operating under the MOE, examines and analyzes the test results and suicide/death data to evaluate the effectiveness of suicide prevention strategies and identifies the characteristics of youth suicide to assess future strategy directions for prevention, maintaining a relatively unified system.

Teen suicide has drawn attention as a major public mental health problem in the past decade. The WHO (2017) reported that the rate of suicide among 15-29 year olds is increas-

This is an Open Access article distributed under the terms of the Creative Commons Attribution Non-Commercial License (https://creativecommons.org/licenses/by-nc/4.0) which permits unrestricted non-commercial use, distribution, and reproduction in any medium, provided the original work is properly cited. ing, suicide being the second leading cause of death. The increase in suicide rate was especially marked in the female teenage group [4], and analysis of the U.S. high school Youth Risk Behavior Surveillance (YRBS) data from 2009 to 2017 revealed a significant correlation between symptoms of depression and increase in suicidal risk only among young females [5], suggesting that personalized intervention based on gender and individual risk factors of at-risk youth is important for effective suicide prevention.

Similar to suicide in adults, the route to suicide in youth is a complex multifactorial interaction including biological, psychological, and societal influences. Moreover, since adolescence is a period of heightened sensitivity to social evaluation, we need to closely monitor how interpersonal relationships, peers, and the socio-cultural environment affect the suicide risk. The school-related risk factors of youth suicide include academic stress, school violence, cyberbullying, and relationship conflict with peers or teachers [6].

For the past ten years since the mental health screening test was expanded nationwide, the role and responsibility of schools in South Korea's student suicide prevention project has gradually increased, from the universal approach of providing mental health promotion services and establishing a safe environment and system for treatment that involves timely detection of suicide risk factors and warning signs and referral to local mental health centers and in-depth investigation of cause of death based on the report of student suicide, including death by teachers, and psychological autopsy by family members. Unfortunately, the rate of teen suicide has not significantly decreased so far [7]. The trend in suicide rate can be interpreted as being in line with the global trend of increasing mental health problems in youth. Moreover, the prolonged global COVID-19 pandemic that started at the end of 2019 is likely to increase students' mental health and suicide risks as they have faced chaotic psycho-social situations, including the effects of social distancing intervention, economic stress, social isolation, barriers to mental health treatment, illness and medical problems, anxiety over national matters [8], as well as school closures and at-home online classes, re- 
stricted daily activities, and poor parenting.

In this special issue, we analyzed the data provided by the Suicide and School Mental Health Institute under MOE. By categorizing the teacher-reported characteristics of students who commit suicide, we have provided a foundation for personalized intervention based on the risk factor characteristics, distinct from the conventional gender and age-specific intervention. In addition, we proposed that among the students who commit or attempt suicide, adolescent suicide survivors have a high suicide risk and providing them with mental health interventions effectively reduces the risk. The report that the ratio of at-risk students receiving treatment at mental health centers as arranged and requested by the school is high is a promising result that indirectly proves that the school suicide prevention strategy has contributed to the promotion of suicide awareness and access to treatment. Moreover, we examined the effect of parenting style on adolescent students' attitudes toward suicide and the indirect effect on suicidal ideation and presented the influence of growth environment and direction of parenting education as distal risk factors. We hope that the papers on this issue will serve as a starting point for not only a microscopic understanding of suicidal youth characteristics but also a macroscopic view of the treatment system and socio-cultural influences to promote active research in the future.

\section{REFERENCES}

1) World Health Organization. National suicide prevention strategies: progress, examples and indicator. Switzerland: World Health Organization;2018.

2) World Health Organization. Adolecsent mental health [cited 2018 Sep 28]. Available from URL: https://www.who.int/mental_ health/maternal-child/adolescent/en/.

3) Suicide and School Mental Health Institute. [cited $2020 \mathrm{Sep} 22$ ]. Available from URL: https://www.ssmhi.com.

4) McLoughlin AB, Gould MS, Malone KM. Global trends in teenage suicide: 2003-2014. QJM 2015;108:765-780.

5) Pontes NMH, Ayres CG, Pontes MCF. Trends in depressive symptoms and suicidality: youth risk behavior survey 2009-2017. Nurs Res 2020;69:176-185.

6) Jung S, Lee D, Park S, Lee K, Kweon YS, Lee EJ, et al. Gender differences in Korean adolescents who died by suicide based on teacher reports. Child Adolesc Psychiatry Ment Health 2019;13:12.

7) Statistics Korea. [cited 2020 Sep 22]. Available from URL: http:// kosis.kr/statisticsList.

8) Reger MA, Stanley IH, Joiner TE. Suicide mortality and coronavirus disease 2019-a perfect storm? JAMA Psychiatry 2020 Apr 10 [Epub ahead of print]. http://doi.org/ 10.1001/jamapsychiatry.2020.1060. 